\title{
Comparison of EBRT and I-125 seed brachytherapy concerning outcome in intermediate-risk prostate cancer
}

\author{
Matthias Moll ${ }^{1}$ (D) Andreas Renner ${ }^{1}$. Christian Kirisits ${ }^{1}$. Christopher Paschen ${ }^{1,2}$ - Alexandru Zaharie ${ }^{1}$. \\ Gregor Goldner ${ }^{1}$
}

Received: 9 April 2021 / Accepted: 1 July 2021 / Published online: 5 August 2021

(c) The Author(s) 2021

\begin{abstract}
Purpose This study's objective was the comparison of external beam radiotherapy (EBRT) and I-125 seed brachytherapy regarding clinical outcome and development of side effects.

Patients and methods In all, 462 localized intermediate-risk prostate cancer patients treated between 2000 and 2019 at our department using either I-125 seed brachytherapy or EBRT with a dose of 74 or 78 Gy were included: 297 patients were treated with EBRT and 165 with seeds. Biochemical no evidence of disease (bNED) rates according to Phoenix definition as well as late gastrointestinal and urogenital side effects (EORTC/RTOG) were assessed.

Results Patients were followed up yearly with a median follow-up of 54 (3-192) months. Observed bNED rates for $74 \mathrm{~Gy}, 78 \mathrm{~Gy}$ and seeds were 87, 92, and $88 \%$ after 5 years and 71, 85, and $76 \%$ after 9 years, respectively. No significant differences were found comparing seeds with $74 \mathrm{~Gy}(p=0.81)$ and $78 \mathrm{~Gy}(p=0.19)$, as well as between 74 and $78 \mathrm{~Gy}$ $(p=0.32)$. Concerning gastrointestinal side effects, EBRT showed significantly higher rates of RTOG grade $\geq 2$ toxicity compared to seeds, but at no point of the follow-up more than $10 \%$ of all patients. However, genitourinary side effects were significantly more prevalent in patients treated with seeds, with 33\% RTOG grade $\geq 2$ toxicity 12 months after treatment. Nevertheless, both types of side effects decreased over time.

Conclusion Favorable intermediate-risk prostate cancer patients can be treated either by external beam radiotherapy (74/78 Gy) or permanent interstitial seed brachytherapy.
\end{abstract}

Keywords Biochemical control $\cdot$ bNED $\cdot$ Toxicity $\cdot$ Side effects $\cdot$ Favourable intermediate risk

\section{Introduction}

Localized primary prostate cancer can be treated via external beam radiotherapy (EBRT) or permanent interstitial seed brachytherapy (BT). Both of these treatment modalities achieve excellent tumor control rates [1-3]. The recommendations and guidelines consider these modalities as equivalent especially for patients with low-risk prostate cancer $[1,2]$. For intermediate-risk patients this strict recommendation to perform seeds BT is missing [1]. Nevertheless, numerous studies evaluating the tumor control rates

Matthias Moll

Matthias.Moll@meduniwien.ac.at

1 Department of Radiation Oncology, Medical University of Vienna, Vienna, Austria

2 Division of Nephrology and Dialysis, Department of Medicine III, Medical University of Vienna, Vienna, Austria after seeds BT included besides low-risk prostate cancer also intermediate-risk patients and reported excellent tumor control rates [4-11]. However, up to now no randomized trial successfully evaluating the effectiveness of seeds BT compared to EBRT has been published so far. The German PREFERE trial [12], coming closest, tried to compare the outcomes of active surveillance, EBRT, BT and prostatectomy in low- and favorable intermediate-risk prostate cancer, but recruited only 345 out of the targeted 7600 patients and was therefore closed, thus, leaving the question unanswered by a prospective study. Our objective with this study is to compare both methods with data acquired from clinical routine patients treated at our department over a period of 20 years. For the evaluation we use data from intermediate-risk prostate cancer patients. We report the results concerning biochemical no evidence of disease (bNED) as well as late gastrointestinal und genitourinary side effects, as increased bNED rates shift the focus on side effects. 


\section{Patients and methods}

The study protocol was approved by the ethical review board of our medical university according to local law regulations (EK no. 1991/2019).

All patients included were treated at our Department of Radiation Oncology and had to meet the following inclusion criteria:

- Intermediate-risk primary prostate cancer as defined by the NCCN classification [13]: Prostate-specific antigen (PSA) between 10 and $20 \mathrm{ng} / \mathrm{ml}$, or Gleason score 7 , or a TNM stage of T2b or T2c,

- Favorable intermediate risk: only one or two risk factors regarding T-stage, PSA or Gleason score,

- Localized cancer with a clinical stage of $\mathrm{cNx} / 0$ and $\mathrm{cMx} / 0$,

- Primary treatment locally limited to the prostate, and

- EBRT patients treated from 2000 to 2015 and I-125 seed BT patients treated from 2004-2019.

Intermediate-risk prostate cancer showing the characteristics above were in general candidates for either EBRT or seeds BT. Both treatment options were discussed with the patient. The final treatment decision was left to the patient.

I-125 seeds were transperineally implanted as a monotherapy. Dose prescription was $145 \mathrm{~Gy}$ for the prostate surrounding isodose according to the TG 137 protocol [14]. The source strength was on average $0.57 \mu \mathrm{Gy} \times \mathrm{m}^{2} / \mathrm{h}$ per seed. All seed applications were performed by one single radiation oncologist. The intervention was performed using spinal anesthesia and patients stayed in hospital for 3 days.

For EBRT, from 2000 to 2009, the prescribed dose was $74 \mathrm{~Gy}$ with $2 \mathrm{~Gy}$ per fraction and patients were treated by three-dimensional (3D) conformal four field box radiotherapy. All patients received a rectal balloon for internal immobilization [15] and the safety margin around the clinical target volume was $10 \mathrm{~mm}$ in all directions for the first $66 \mathrm{~Gy}$ followed by $8 \mathrm{~Gy}$ with reduced dorsal margin of $5 \mathrm{~mm}$.

For EBRT from 2010 to 2015 dose was escalated to 78 Gy with $2 \mathrm{~Gy}$ per fraction using either 3D conformal radiotherapy or volume modulated arc therapy (VMAT) from 2013 onwards. Again, all patients received a rectal balloon and in addition prior to radiotherapy gold marker fiducials were implanted. Due to the long time frame of our study, safety margins varied over time. The safety margin around the clinical target volume was $5 \mathrm{~mm}$ in all directions with gold marker fiducials, $7 \mathrm{~mm}$ in all directions without for $78 \mathrm{~Gy}$, and $10 \mathrm{~mm}$ in the $74 \mathrm{~Gy}$ group for the first $66 \mathrm{~Gy}$ and $5 \mathrm{~mm}$ for the last $8 \mathrm{~Gy}$.

Hormonal therapy was recommended for patients receiving EBRT for a duration of 6 months. However, the prescription of hormonal therapy was in the hands of the refer- ring urologists and a certain proportion of patients refused hormonal therapy.

bNED failure was defined as PSA nadir $+2 \mathrm{ng} / \mathrm{ml}$ using the Phoenix criteria [16]. Follow-up after treatment was scheduled after 3 months, 12 months, and every year from that point on. For each follow-up, gastrointestinal and genitourinary side effects were prospectively assessed using RTOG grading [17], and PSA levels were documented. All measures of time were calculated from the last day of radiotherapy.

Statistical analysis was performed using GraphPad Prism 9 (GraphPad Software, San Diego, CA, USA) and SPSS 26 (IBM, Armonk, NY, USA). All statistical tests were two-sided and a $p$-level $<0.05$ was considered statistically significant. The Kaplan-Meier method was used to estimate bNED rates. The resulting curves were compared using the log-rank test. The univariate Cox regression model included the following variables: T-stage, PSA, Gleason score, androgen deprivation therapy (ADT) and treatment modality. For all analyses, Gleason score $(0=" 2-6 "$ vs. $1=" 7 ")$, T-stage $(0=$ "T1a-c and $2 \mathrm{a}$ " vs. $1=$ "T2b/c"), hormonal therapy $(0=$ "no" vs. $1=$ "yes"), risk groups $(0=$ "one risk factor", $1=$ " 2 risk factors") and dose $(0=$ "74 Gy" vs $1=$ "78 Gy" and $2=$ "seeds") were treated as categorical variables with the lowest category serving as reference category, while PSA was treated as an ordinal variable. Side effects were analyzed using the Mann-Whitney U test. Internal testing regarding the difference between 74 and 78 Gy over the course of 10 years was performed. Within this time span, the only significant differences we found were 36 and 72 months in concerning gastrointestinal side effects. Therefore, we merged both EBRT groups. Side effects are arranged in a group with RTOG grade 0 and 1 and another one with RTOG grade 2 and higher. Thereby, we aim to provide a better overview of the level of occurrence of clinically relevant side effects.

\section{Results}

Our retrospective analysis included 462 primary intermediate-risk prostate cancer patients with a median follow-up of 54 (3-192) months: 297 patients received EBRT, 185 with a total dose of $74 \mathrm{~Gy}$ and 112 with a total dose of $78 \mathrm{~Gy}$. Furthermore, 165 patients were treated with seeds. Further relevant patient characteristics are summarized in Table 1.

Concerning the treatment modalities, most notable differences are the lower rate of Gleason score 6 patients and the lower percentage of ADT in BT patients compared to EBRT. The median follow-up was longer for patients treated with $74 \mathrm{~Gy}$ (60 months), as the application of $78 \mathrm{~Gy}$. Furthermore, $47 \%$ of all patients had a followup of at least 60 months. As of 2019, $60 \%$ of all patients 
Table 1 Patient characteristics

\begin{tabular}{|c|c|c|c|c|c|c|}
\hline & 74 Gy & $\%$ & $78 \mathrm{~Gy}$ & $\%$ & $\begin{array}{l}\text { I-125 } \\
\text { seeds }\end{array}$ & $\%$ \\
\hline$n=$ & 185 & & 112 & & 165 & \\
\hline \multicolumn{7}{|l|}{ T-stage } \\
\hline $1 a / b$ & 10 & 5 & 5 & 4 & 0 & 0 \\
\hline $1 c$ & 93 & 50 & 57 & 51 & 113 & 68 \\
\hline $2 a$ & 29 & 16 & 13 & 12 & 29 & 18 \\
\hline $2 b / c$ & 53 & 29 & 37 & 33 & 23 & 14 \\
\hline \multicolumn{7}{|l|}{ iPSA in $\mathrm{ng} / \mathrm{ml}$} \\
\hline Min & 1.98 & - & 1.5 & - & 1.7 & - \\
\hline $\operatorname{Max}$ & 19.90 & - & 19.9 & - & 19.0 & - \\
\hline Median & 10.2 & - & 7.4 & - & 7.2 & - \\
\hline \multicolumn{7}{|l|}{ Gleason score } \\
\hline$<6$ or 6 & 94 & 51 & 47 & 42 & 58 & 35 \\
\hline $7 a$ & 36 & 19 & 40 & 36 & 70 & 42 \\
\hline $7 b$ & 18 & 10 & 24 & 21 & 26 & 16 \\
\hline $\begin{array}{l}7 \text { not otherwise } \\
\text { specified }\end{array}$ & 37 & 20 & 1 & 1 & 11 & 7 \\
\hline \multicolumn{7}{|l|}{ ADT } \\
\hline Yes & 119 & 64 & 40 & 36 & 31 & 19 \\
\hline $\begin{array}{l}\text { Median dura- } \\
\text { tion in months }\end{array}$ & 10 & - & 11 & - & 7 & 4 \\
\hline \multicolumn{7}{|c|}{ Age during treatment in years } \\
\hline Min & 54 & - & 47 & - & 49 & - \\
\hline $\operatorname{Max}$ & 85 & - & 83 & - & 86 & - \\
\hline Median & 73 & - & 74 & - & 69 & - \\
\hline \multicolumn{7}{|l|}{ Follow-up } \\
\hline Min & 3 & - & 3 & - & 3 & - \\
\hline $\operatorname{Max}$ & 192 & - & 108 & - & 166 & - \\
\hline $\begin{array}{l}\text { Median in } \\
\text { months }\end{array}$ & 60 & - & 48 & - & 50 & - \\
\hline \multicolumn{7}{|l|}{ Technique } \\
\hline Seeds & 0 & 0 & 0 & 0 & 165 & 100 \\
\hline $3 D$ conformal & 183 & 99 & 66 & 59 & 0 & 0 \\
\hline VMAT or IMRT & 2 & 1 & 46 & 41 & 0 & 0 \\
\hline \multicolumn{7}{|l|}{ Risk group factor } \\
\hline 1 & 131 & 71 & 85 & 76 & 155 & 94 \\
\hline 2 & 54 & 29 & 27 & 24 & 10 & 6 \\
\hline
\end{tabular}

$A D T$ androgen deprivation therapy, IMRT intensity-modulated radiation therapy, VMAT volumetric modulated arc therapy

were lost to follow-up.bNED rates for $74 \mathrm{~Gy}, 78 \mathrm{~Gy}$, and seeds are displayed in Fig. 1. For 74 Gy, bNED rates after 5 and 9 years were $87 \%$ and $71 \%$. The latest reported bNED failure occurred after 126 months. In the $78 \mathrm{~Gy}$ group, corresponding bNED rates were $92 \%$ after 5 years and $85 \%$ after 9 years, respectively. For seeds, the bNED rates were $88 \%$ after 5 years and $76 \%$ after 9 years. Comparing the Kaplan-Meier curves, 74 Gy vs 78 Gy and seeds vs 74 or $78 \mathrm{~Gy}$ showed no significance ( $p=0.32,0.81$, and 0.19 , respectively).

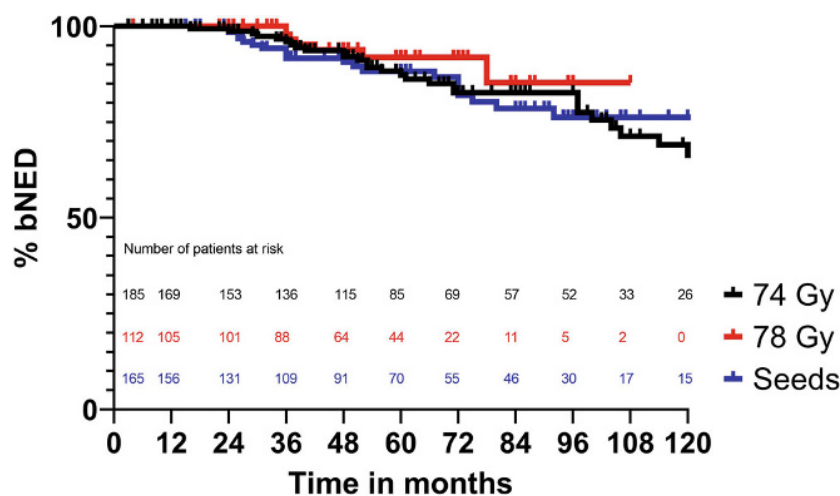

Fig. 1 bNED after either EBRT (with 74 or $78 \mathrm{~Gy}$ ) or seeds. $P$ value $=0.32$ comparing 74 and $78 \mathrm{~Gy}, p=0.81$ comparing $74 \mathrm{~Gy}$ and seeds, $p=0.19$ comparing $78 \mathrm{~Gy}$ and seeds

Table 2 Univariate analysis of bNED

\begin{tabular}{lllll}
\hline & $p$ & HR & $\begin{array}{l}\text { Lower } \\
95 \% \text { CI }\end{array}$ & $\begin{array}{l}\text { Upper } \\
95 \% \text { CI }\end{array}$ \\
\hline T2b/c vs T1a-c and 2a & 0.10 & 1.60 & 0.92 & 2.78 \\
$\begin{array}{l}\text { Gleason score 7 vs 6 } \\
\text { and <6 }\end{array}$ & 0.89 & 0.96 & 0.57 & 1.63 \\
Initial PSA & 0.43 & 1.03 & 0.96 & 1.10 \\
2 risk factors vs 1 & 0.11 & 1.60 & 0.90 & 2.86 \\
ADT applied & 0.04 & 0.57 & 0.33 & 0.98 \\
Age & 0.18 & 0.97 & 0.93 & 1.01 \\
78 Gy vs 74 Gy & 0.22 & 0.59 & 0.25 & 1.37 \\
Seeds vs 74Gy & 0.81 & 0.93 & 0.53 & 1.65 \\
Seeds vs 78 Gy & 0.20 & 1.76 & 0.74 & 4,22 \\
\hline
\end{tabular}

$A D T$ androgen deprivation therapy

Table 2 displays the results of the univariate analysis. Significant influences regarding the bNED outcome were only detectable in patients receiving ADT. This result was also confirmed in a log-rank test, comparing all patients who received ADT with those who did not $(p=0.04)$.

For further analysis, we compared patients who received ADT vs patients who did not within each group. For 74 and $78 \mathrm{~Gy}$, no significant differences were found $(p=0.91$ and 0.23 ). For BT, we discovered a significant advantage for ADT $(p=0.005)$, without a single bNED failure for patients receiving ADT over a median of 84 months, but within a small collective of 31 patients. As we discovered ADT as the only significant factor in our univariate analysis, no further multivariate analyses were performed.

Maximum late side effects at any point during treatment and follow-up are displayed in Table 3. Overall, all our subgroups tolerated the treatment well, with no more than $34 \%$ of any grade 2 toxicity in any group. Furthermore, patients receiving EBRT report in $61 \%$ of the cases no gastrointestinal and in 50\% no genitourinary side effects. For seeds, $65 \%$ of all patients report no gastrointestinal side effects, but only $10 \%$ report no genitourinary side effects. 


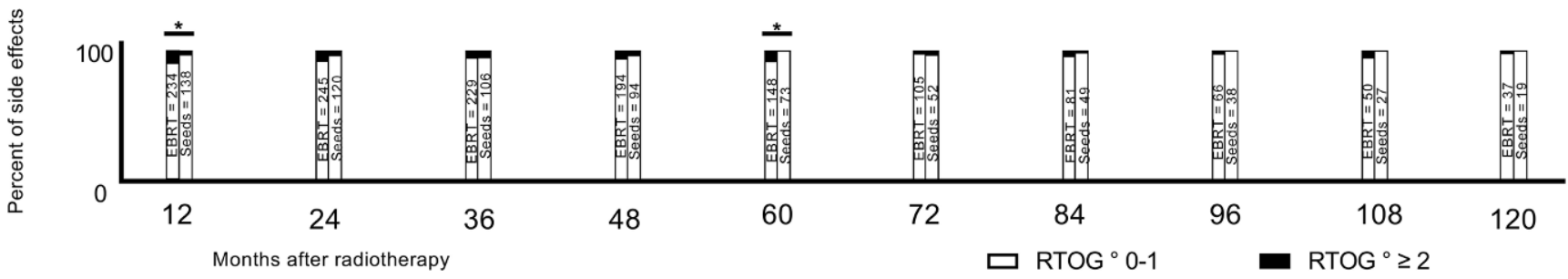

Fig. 2 Development of gastrointestinal side effects after treatment with EBRT or seeds over a follow-up period of 120 months $(* p<0.05$, $* * p<0.01, * * * p<0.001)$

There were 3 patients suffering from grade 4 toxicity reported. One patient treated with 78 Gy developed a fistula and needed a transient stoma that was surgically removed after 6 months. Two patients treated with seeds developed RTOG grade 4 genitourinary toxicity in the form of urinary retention that required operative urological intervention. Comparing the data regarding side effects from grade 0 to 4 , there was a significant difference between $74 \mathrm{~Gy}$ and 78 Gy groups concerning maximum genitourinary side effects $(p=0.04)$. No difference was found for maximum gastrointestinal side effects in EBRT $(p=0.14)$. Comparing EBRT with seeds, we observed a borderline significant difference $(p=0.06)$ regarding maximum late gastrointestinal and a significant difference regarding genitourinary toxicity ( $p=0.06$ and $p<0.001$, respectively).

The course of side effects over a follow-up period of 120 months is displayed in Figs. 2 and 3. Internal testing regarding the difference between 74 and $78 \mathrm{~Gy}$ over the course of 10 years was performed. Side effects are arranged in a group with RTOG grade 0 and 1 and another one with RTOG grade 2 and higher. Thereby, we aim to provide a better overview of the level of occurrence of clinically relevant side effects.

For the first and fifth year of follow-up, we observed a significantly higher level of gastrointestinal RTOG grade $\geq 2$ toxicity in patients treated with EBRT. Nevertheless, the highest rate of RTOG grade $\geq 2$ toxicity was only observed in $10 \%$ of patients treated with EBRT after 12 months of follow-up. From this point on, higher levels of gastrointestinal toxicity declined over time and were almost gone after 120 months of follow-up.
Genitourinary RTOG grade $\geq 2$ toxicity was significantly higher in patients treated with seeds up to a maximum of $33 \%$ after 12 months of follow-up. While, just like gastrointestinal side effects, also declining over time, $11 \%$ of all seeds patients reported RTOG grade $\geq 2$ toxicity after 120 months of follow-up. For EBRT, RTOG grade $\geq 2$ toxicity alternated between $3 \%$ and $13 \%$ of all patients without a decline over time. Differences regarding the number of patients at risk between bNED rate and toxicity are due to lack of documented PSA and toxicity.

\section{Discussion}

Intermediate-risk prostate cancer can be equally effectively treated either via radical prostatectomy or EBRT [13, 18-20]. The role of BT in these patients is seen critically, especially in the German S3 guideline, although several studies show good biochemical control rates in intermediate-risk prostate cancer patients [4-11, 21]. A strict recommendation for permanent interstitial low dose rate (LDR)BT is limited to low-risk patients. Up to now, no randomized trial comparing LDR-BT versus EBRT versus radical prostatectomy was successfully completed-regardless of patients' prostate cancer risk. The ratio to restrict the recommendation for LDR-BT to the low-risk group is their excellent tumor control rates. Nevertheless, various studies reporting about LDR-BT also included beside low-risk patients, intermediate-risk patients with reliable proportion and respectable tumor control rates. Due to the lack of randomized trials, retrospective analysis including a sufficient

Table 3 Maximum of late gastrointestinal and genitourinary side effects

\begin{tabular}{|c|c|c|c|c|c|c|c|c|}
\hline \multirow{2}{*}{$\begin{array}{l}\text { Maximum of } \\
\text { RTOG }\end{array}$} & \multicolumn{4}{|c|}{ Gastrointestinal side effects } & \multicolumn{4}{|c|}{ Genitourinary side effects } \\
\hline & $74 \mathrm{~Gy}$ & $78 \mathrm{~Gy}$ & EBRT & Seeds & $74 \mathrm{~Gy}$ & $78 \mathrm{~Gy}$ & EBRT & Seeds \\
\hline Grade 0 & $64 \%$ & $55 \%$ & $61 \%$ & $65 \%$ & $54 \%$ & $43 \%$ & $50 \%$ & $10 \%$ \\
\hline Grade 1 & $15 \%$ & $21 \%$ & $17 \%$ & $26 \%$ & $23 \%$ & $23 \%$ & $23 \%$ & $18 \%$ \\
\hline Grade 2 & $19 \%$ & $22 \%$ & $20 \%$ & $8 \%$ & $17 \%$ & $28 \%$ & $21 \%$ & $67 \%$ \\
\hline Grade 3 & $2 \%$ & $2 \%$ & $2 \%$ & $0 \%$ & $6 \%$ & $6 \%$ & $6 \%$ & $4 \%$ \\
\hline Grade 4 & $0 \%$ & $1 \%$ & $0 \%$ & $0 \%$ & $0 \%$ & $0 \%$ & $0 \%$ & $1 \%$ \\
\hline$n$ & 185 & 111 & 296 & 165 & 185 & 111 & 296 & 165 \\
\hline
\end{tabular}




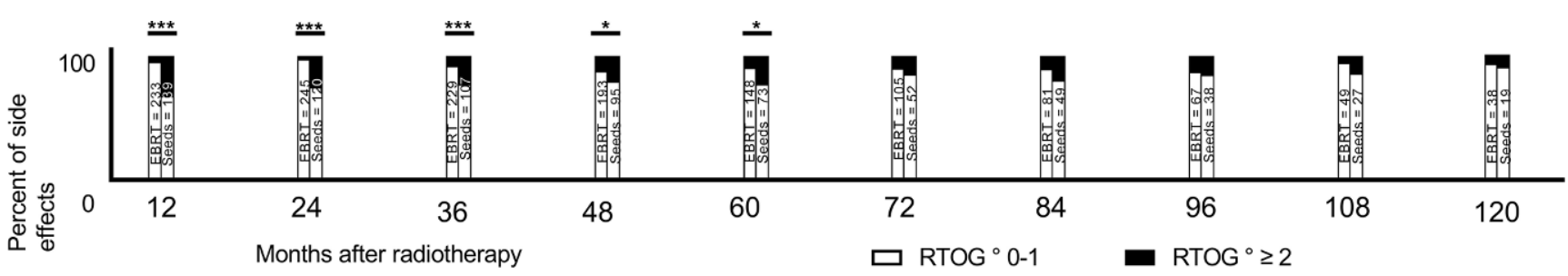

Fig. 3 Development of genitourinary side effects after treatment with EBRT or seeds over a follow-up period of 120 months $\left({ }^{*} p<0.05\right.$, ${ }^{* *} p<0.01$, $* * * p<0.001$ )

proportion of patients treated within a center of excellence by EBRT with a dose corresponding to the dose recommended within the guidelines (at least $74 \mathrm{~Gy}$ ) or LDR-BT are important.

Concerning our bNED rates in seeds patients, the results of $88 \%$ bNED after 5 years and $76 \%$ after 10 years are within range of rates reported in the literature. A comparison of EBRT and seed BT published by Goldner et al. in 2012 [4] showed a bNED rate after 5 years of $75 \%$ for EBRT with $74 \mathrm{~Gy}$ and $81 \%$ for seeds. Now, we are able to display a bNED rate of $87 \%$ after 5 years for $74 \mathrm{~Gy}$ and $88 \%$ for seeds, respectively. With this data, we are able to recreate the lack of significant differences between $74 \mathrm{~Gy}$ and seeds for our selected intermediate-risk prostate cancer patients. Within our analysis $78 \mathrm{~Gy}$ provides a slightly better biochemical control as the other treatment modalities, although without a significant difference. This might be partly due to the fact that $21 \%$ our $78 \mathrm{~Gy}$ patients had a Gleason score of $7 \mathrm{~b}$, compared to $10 \%$ and $16 \%$ of our $74 \mathrm{~Gy}$ and seeds patients, respectively. Regarding the univariate analysis, we could not confirm T-stage, Gleason score, and iPSA as significant factors, while ADT was confirmed.

The reported bNED rates after seeds for intermediaterisk in the literature range from around $80 \%$ to $90 \%$ [4, 6, $7,11]$ and above $[5,10]$ after 5 years and about $55 \%$ [4] over 75\% [11] to around 90\% [7] after 10 years. Regarding the $74 \mathrm{~Gy}$ group, our bNED rate is $87 \%$ after 5 years and $66 \%$ after 10 years. These results are better than the reported bNED rates in intermediate-risk patients after 8 years in a large Australian study [22] with $66 \%$. It is, however, noteworthy that this study also used doses with a total of $70 \mathrm{~Gy}$, which is known to be insufficient in intermediaterisk [4] and even low-risk [23, 24]. The reported bNED rate of the CHHiP trial [25] with around $88 \%$ after 5 years in the $74 \mathrm{~Gy}$ group is pretty close to our results, although the trial included also low- and high-risk patients and did administer ADT in $97 \%$ of the cases, which in our case was an important factor regarding bNED. The MRC RT01 trial [26] showed 5 year bNED rates of $71 \%$ and 10 year bNED of $55 \%$ with $3 \mathrm{D}$ conformal radiotherapy and inclusion of low-, intermediate-, and high-risk patients. The results of the 78 Gy group with $92 \%$ bNED rate after 5 years and $85 \%$ after 9 years are slightly worse than the outcomes reported by Pasalic et al. [27]. However, caution is advised, as only 2 patients in the $78 \mathrm{~Gy}$ group have a follow-up this long. Compared to the free rate of $74 \%$ after 5 years reported by Peeters et al. [28], our patients show higher bNED rates, most likely due to the fact that Peeters included a large number of high-risk patients. In addition, failure-free rates were defined using the ASTRO definition.

Regarding late side effects, we observed reduced genitourinary side effects and increased gastrointestinal side effects for EBRT in comparison to seeds. This resembles the results in our low-risk collective [3]. For maximum side effects, we noticed a significant increase for late genitourinary side effects in patients treated with $78 \mathrm{~Gy}$ compared to $74 \mathrm{~Gy}$. This might be partly explained due to our $78 \mathrm{~Gy}$ patient collective being the oldest one with a median age of 74 years, compared to 73 years in the $74 \mathrm{~Gy}$ group and 69 years in the seeds group. Therefore, late GU toxicities for seeds patients might also be underestimated. For late gastrointestinal side effects, we were not able to record a significant difference, possibly due to reduced dorsal PTVs in patients treated with $78 \mathrm{~Gy}$. The alternating but not decaying amount of genitourinary grade 2 toxicity in EBRT might be the result of our patient collective getting older and therefore intrinsically developing more genitourinary problems that might be classified as toxicity.

The main weakness of our study is the retrospective nature and the high proportion of patients with missing followup, resulting in median follow-up rates of only 54 months. However, in terms of biochemical control, the follow-up is sufficiently long. Another potential weakness is the choice of treatment modality by the patient, leading to a possible selection bias. On the other hand, our study shows several strengths. As a monocentric study, reporting of side effects is similar of the observed period. Having all patients with seeds treated by only one radiation oncologist allows to assume a high level of quality in treatment, as displayed by Nakamura et al. [29]. Furthermore, our collected data originated from daily clinical practice. 


\section{Conclusion}

Regarding bNED rates, no significant difference between seed brachytherapy and external beam radiotherapy ( 74 or $78 \mathrm{~Gy}$ ) could be detected in our selected patient collective. Concerning late side effects, less genitourinary, and higher gastrointestinal side effects are observed in EBRT.

Acknowledgements Special thanks to Marion Moll for English language editing.

Funding Open access funding provided by Medical University of Vienna.

\section{Declarations}

Conflict of interest M. Moll, A. Renner, C. Kirisits, C. Paschen, A. Zaharie and G. Goldner declare that they have no competing interests.

Ethical standards All procedures performed in studies involving human participants or on human tissue were in accordance with the ethical standards of the institutional (ethical review board of our medical university EK Nr. 1991/2019) and/or national research committee and with the 1975 Helsinki declaration and its later amendments or comparable ethical standards.

Open Access This article is licensed under a Creative Commons Attribution 4.0 International License, which permits use, sharing, adaptation, distribution and reproduction in any medium or format, as long as you give appropriate credit to the original author(s) and the source, provide a link to the Creative Commons licence, and indicate if changes were made. The images or other third party material in this article are included in the article's Creative Commons licence, unless indicated otherwise in a credit line to the material. If material is not included in the article's Creative Commons licence and your intended use is not permitted by statutory regulation or exceeds the permitted use, you will need to obtain permission directly from the copyright holder. To view a copy of this licence, visit http://creativecommons.org/licenses/by/4. $0 /$.

\section{References}

1. Leitlinienprogramm Onkologie (2021) S3-Leitlinie Prostatakarzinom, Version 6.0, 05/2021. https://www.leitlinienprogrammonkologie.de/mwg-internal/de5fs23hu73ds/progress?id=6EduY9K 1iK09VZIlf8dOvGrff8piCbbjGVH9Qi0SqOo. Accessed 16 June 2021

2. Mohler JL et al (2019) NCCN clinical practice guidelines in oncology_-prostate cancer version 4.2019

3. Moll M, Paschen C, Zaharie A, Berndl F, Goldner G (2020) Treatment of low-risk prostate cancer: a retrospective study with 477 patients comparing external beam radiotherapy and I- 125 seeds brachytherapy in terms of biochemical control and late side effects. Strahlenther Onkol. https://doi.org/10.1007/s00066-020-01657-1

4. Goldner $\mathrm{G}$ et al (2012) Comparison between external beam radiotherapy $(70 \mathrm{~Gy} / 74 \mathrm{~Gy})$ and permanent interstitial brachytherapy in 890 intermediate risk prostate cancer patients. Radiother Oncol 103:223-227

5. Frank SJ et al (2018) Prospective phase 2 trial of permanent seed implantation prostate brachytherapy for intermediate-risk localized prostate cancer: efficacy, toxicity, and quality of life outcomes. Int J Radiat Oncol Biol Phys 100:374-382
6. Munro NP et al (2010) Outcomes from Gleason 7, intermediate risk, localized prostate cancer treated with Iodine-125 monotherapy over 10 years. Radiother Oncol 96:34-37

7. Matzkin H, Chen J, Agai R, Ziv-Baran T, Mabjeesh NJ (2019) Long-term biochemical progression-free survival following brachytherapy for prostate cancer: further insight into the role of shortterm androgen deprivation and intermediate risk group subclassification. PLoS ONE 14:1-16

8. Cosset JM et al (2008) Selecting patients for exclusive permanent implant prostate brachytherapy: the experience of the paris Institut Curie/Cochin Hospital/Necker Hospital Group on 809 patients. Int J Radiat Oncol Biol Phys 71:1042-1048

9. Martell K et al (2019) Results of 15 Gy HDR-BT boost plus EBRT in intermediate-risk prostate cancer: analysis of over 500 patients. Radiother Oncol 141:149-155

10. Guimond E et al (2019) Impact of a dominant intraprostatic lesion (DIL) boost defined by sextant biopsy in permanent I-125 prostate implants on biochemical disease free survival (bDFS) and toxicity outcomes. Radiother Oncol 133:62-67

11. Prada PJ et al (2018) Long-term outcomes in patients younger than 60 years of age treated with brachytherapy for prostate cancer. Strahlenther Onkol 194:311-317

12. Wiegel $\mathrm{T}$ et al (2021) Results of a randomized trial of treatment modalities in patients with low or early-intermediate risk prostate cancer (PREFERE trial). J Cancer Res Clin Oncol 147:235-242

13. Mohler JL et al (2019) NCCN clinical practice guidelines in oncology-prostate cancer, version 2.2019 short. J Natl Compr Canc Netw 17:479-505

14. Nath $\mathrm{R}$ et al (2009) AAPM recommendations on dose prescription and reporting methods for permanent interstitial brachytherapy for prostate cancer: report of Task Group 137. Med Phys 36(11):5310-5322

15. Wachter S et al (2002) The influence of a rectal balloon tube as internal immobilization device on variations of volumes and dosevolume histograms during treatment course of conformal radiotherapy for prostate cancer. Int J Radiat Oncol Biol Phys 52:91-100

16. Roach $M$ et al (2006) Defining biochemical failure following radiotherapy with or without hormonal therapy in men with clinically localized prostate cancer: recommendations of the RTOGASTRO Phoenix Consensus Conference. Int J Radiat Oncol Biol Phys 65:965-974

17. Cox JD, Stetz J, Pajak TF (1995) Toxicity criteria of the Radiation Therapy Oncology Group (RTOG) and the European Organization for Research and Treatment of Cancer (EORTC). Int J Radiat Oncol Biol Phys 31:1341-1346

18. Leitlinienprogramm Onkologie (2019) S3-Leitlinie Prostatakarzinom. Version 5.1, 05/2019

19. Hamdy FC et al (2016) 10-year outcomes after monitoring, surgery, or radiotherapy for localized prostate cancer. N Engl J Med $375: 1415-1424$

20. Taguchi S et al (2019) Robot-assisted radical prostatectomy versus volumetric modulated arc therapy: comparison of front-line therapies for localized prostate cancer. Radiother Oncol 140:62-67

21. Hinnen KA et al (2010) Loose seeds versus stranded seeds in I-125 prostate brachytherapy: differences in clinical outcome. Radiother Oncol 96:30-33

22. de Leon JF et al (2019) Long-term outcomes in 1121 Australian prostate cancer patients treated with definitive radiotherapy. J Med Imaging Radiat Oncol 63:116-123

23. Goldner $G$ et al (2012) Comparison of seed brachytherapy or external beam radiotherapy ( $70 \mathrm{~Gy}$ or $74 \mathrm{~Gy}$ ) in 919 low-risk prostate cancer patients. Strahlenther Onkol 188:305-310

24. Kupelian PA et al (2004) Radical prostatectomy, external beam radiotherapy $<72 \mathrm{~Gy}$, external beam radiotherapy $\geq 72 \mathrm{~Gy}$, permanent seed implantation, or combined seeds/external beam radiotherapy 
for stage T1-T2 prostate cancer. Int J Radiat Oncol Biol Phys $58: 25-33$

25. Dearnaley D et al (2016) Conventional versus hypofractionated high-dose intensity-modulated radiotherapy for prostate cancer: 5 -year outcomes of the randomised, non-inferiority, phase $3 \mathrm{CHHiP}$ trial. Lancet Oncol 17:1047-1060

26. Dearnaley DP et al (2007) Escalated-dose versus standard-dose conformal radiotherapy in prostate cancer: first results from the MRC RT01 randomised controlled trial. Lancet Oncol 8:475-487

27. Pasalic D et al (2019) Dose escalation for prostate adenocarcinoma: a long-term update on the outcomes of a phase 3 , single institution randomized clinical trial. Int J Radiat Oncol Biol Phys 104:790-797
28. Peeters STH et al (2006) Dose-response in radiotherapy for localized prostate cancer: results of the Dutch multicenter randomized phase III trial comparing $68 \mathrm{~Gy}$ of radiotherapy with $78 \mathrm{~Gy}$. J Clin Oncol 24:1990-1996

29. Nakamura $K$ et al (2019) Institutional patient accrual volume and the treatment quality of I-125 prostate seed implantation in a Japanese nationwide prospective cohort study. Strahlenther Onkol 195:412-419 